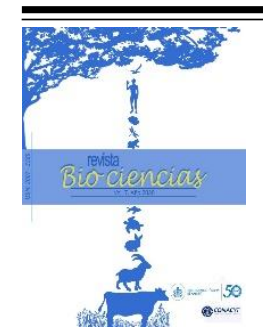

Revista Bio Ciencias

https://revistabiociencias.uan.edu.mx

ISSN 2007-3380
Memorias del 3er

Coloquio de

Nutrigenómica y

Biotecnología Acuícola

2020

\title{
Uso de compuestos funcionales en dietas bajas en harina de pescado para totoaba.
} Fuentes-Quesada, J.P.

Stolt Sea Farm, A Cuiña-Cervo 154 Polígono Industrial, Cervo 27890, Spain.

*E-mail: pablofuentesq@gmail.com

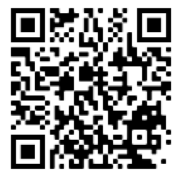

Cite this paper/Como citar este artículo: Fuentes-Quesada, J. P. (2021). Uso de compuestos funcionales en dietas bajas en harina de pescado para totoaba. Revista Bio Ciencias 8: (Suppl) Memorias del 3er Coloquio de Nutrigenómica y Biotecnología Acuícola 2020 (CONYBA) e1180. http://doi.org/10.15741/revbio.08Suppl.e1180

\section{Resumen}

Hoy en día, la inclusión de ingredientes de origen marino en la formulación de dietas para peces marinos es cada vez menor, debido a su alto costo y como medida de sostenibilidad del recurso en los mares. Ante esta necesidad, se ha incrementado el nivel de ingredientes de origen terrestre, en especial las harinas vegetales, que tienen un menor costo y se producen en grandes volúmenes. Sin embargo, ciertos factores antinutricionales que contienen las harinas vegetales puede provocar respuestas adversas en el crecimiento, eficiencia alimenticia, sistema inmune, morfología digestiva y microbiota intestinal de los peces, afectando de manera especial a las especies carnívoros. Como alternativa para mejorar el desempeño de las dietas en peces, en los últimos años se han realizado numerosos estudios utilizando compuestos de origen biológico que tienen tener un efecto selectivo sobre una 0 varias funciones del organismo y que le confieren un beneficio a la salud del individuo, a los cuales se les ha llamado compuestos funcionales.

\footnotetext{
Abstract

Today, the inclusion of ingredients of marine origin in the formulation of diets for marine fish is decreasing, due to its high cost and as a measure of the sustainability of the resource. Given this need, the level of ingredients of terrestrial origin has been increased, especially vegetable meals, which are produced in large volumes at lower cost. However, certain antinutritional factors that vegetable meals have can cause adverse effects on the growth, immune system, and intestinal microbiota of fish, especially affecting carnivorous species. As an alternative to improve the performance of fish diets, in recent years there have been numerous studies using compounds that have a selective effect on one or several functions of the organism, and that confers a benefit to the health of
}

the individual, which have been called functional compounds.

\section{Introducción}

Una adecuada nutrición es un factor clave para garantizar un crecimiento óptimo y una salud estable en los peces de cultivo. Sin embargo, las formulaciones de las dietas para peces marinos cada vez incluyen una mayor variedad de materias primas, debido a las restricciones en el uso de ingredientes de origen marino (IOM) por su alta demanda que ha incrementado su costo. En el caso de las dietas de especies emergentes, como es el caso de la totoaba (Totoaba macdonaldi), una especie endémica y carnívora del Golfo de California, las formulaciones utilizadas al inicio son adaptadas de otras especies, con la limitante que pueden no suplir todos los requerimientos específicos y que las materias primas usadas pueden contener factores antinutricionales como ocurre en las harinas de origen vegetal.

Los efectos adversos en el desempeño, supresión de la salud y alteraciones en la estructura y microbiota intestinal de peces provocadas por el uso de ingredientes vegetales han sido reportados en varias especies (Krogdahl et al., 2010; Gu et al., 2016; Bonvini et al., 2018), incluyendo la totoaba (Fuentes-Quesada et al., 2018), lo que ha llevado a restringir o limitar su inclusión en las dietas.

Ante esta situación, estudios en los últimos años realizados en especies terrestres han encontrado compuestos, que pueden ser nutrientes o no, que confieren beneficios en el rendimiento productivo y la salud intestinal de los organismos que lo consumen (Øverland et al., 2019).

Los beneficios potenciales y que pueden ser posibles alternativas a los antibióticos han generado un gran interés en el uso de estos compuestos funcionales en la acuicultura para mejorar la productividad y salud de los animales de cultivo (Pelusio et al., 2020), especialmente, 
cuando las dietas incluyen ingredientes de origen vegetal.

Dentro de los compuestos funcionales podemos encontrar aminoácidos ácidos grasos -esenciales o no-, ácidos grasos de cadena corta, carotenoides, pigmentos, péptidos bioactivos, ácidos orgánicos, pre y probióticos, compuestos activos de plantas, nucleótidos, entre otros.

El uso de este tipo de compuestos funcionales como estrategia nutricional cuando se formulan dietas con ingredientes vegetales en peces han demostrado mejorar el crecimiento, la utilización del alimento, respuesta inmune, la integridad intestinal y conservar una microbiota funcional (Pereira et al., 2017; Guerreiro et al.,2017; Chen et al., 2018).

Ante estos hallazgos y debido a los efectos adversos de harinas vegetales en el crecimiento e integridad intestinal de la totoaba, se evaluó la utilización de dos compuestos funcionales, un fructano como prebiótico y un aminoácido condicional como la glutamina en dietas bajas en harina de pescado con inclusión de harina vegetal sobre el efecto en el crecimiento, eficiencia alimenticia y salud intestinal.

\section{Materiales y métodos}

Diseño experimental 1: formulación de las dietas Cuatro dietas formuladas isoproteícas (51\%) e isolipídicas (12\%), la dieta control la fuente proteica fue solo la harina de pescado (FM, por sus siglas en inglés). En la segunda dieta se reemplazó la FM en un $67 \%$ por harina de subproducto de ave (PBM, por sus siglas en inglés) manteniendo una proporción 2:1. En la tercera dieta se agregó 24\% de harina de soya (SBM, por sus siglas en inglés) y se redujo la PBM y FM en la misma proporción 2:1. La cuarta dieta tiene la misma formulación que la tercera dieta, pero se añadió un $2 \%$ de fructano obtenido de la planta de agave (AGA).

\section{Experimento 1}

Tabla 1. Crecimiento y utilización del alimento de la totoaba a los 44 días de alimentación.

\begin{tabular}{lccccc} 
& FM & PBM & SBM & 2\% AGA & P value \\
\hline Initial weight (g) & $60.7 \pm 1.5$ & $60.7 \pm 2.5$ & $61.0 \pm 2.0$ & $60.7 \pm 1.5$ & 0.995 \\
Final weight $(\mathrm{g})$ & $200.2 \pm 3.0^{\mathrm{ab}}$ & $196.7 \pm 1.1^{\mathrm{b}}$ & $195.0 \pm 3.4^{\mathrm{b}}$ & $207.1 \pm 3.8^{\mathrm{a}}$ & 0.005 \\
$\mathrm{TGC}^{1}$ & $1.85 \pm 0.01^{\mathrm{ab}}$ & $1.82 \pm 0.05^{\mathrm{ab}}$ & $1.79 \pm 0.05^{\mathrm{b}}$ & $1.91 \pm 0.02^{\mathrm{a}}$ & 0.023 \\
$\mathrm{WG}^{2}(\mathrm{~g})$ & $139.6 \pm 1.6^{\mathrm{ab}}$ & $136.0 \pm 2.9^{\mathrm{ab}}$ & $134.0 \pm 3.7^{\mathrm{b}}$ & $146.4 \pm 2.6^{\mathrm{a}}$ & 0.003 \\
$\mathrm{RWG}^{3}(\%)$ & $230.1 \pm 3.9^{\mathrm{ab}}$ & $224.6 \pm 13.9^{\mathrm{ab}}$ & $219.9 \pm 11.1^{\mathrm{b}}$ & $241.4 \pm 4.4^{\mathrm{a}}$ & 0.045 \\
$\mathrm{DWG}^{4}(\mathrm{~g})$ & $3.17 \pm 0.04^{\mathrm{ab}}$ & $3.09 \pm 0.01^{\mathrm{b}}$ & $3.05 \pm 0.08^{\mathrm{b}}$ & $3.33 \pm 0.06^{\mathrm{a}}$ & 0.003 \\
$\mathrm{FCR}^{5}$ & $0.85 \pm 0.01^{\mathrm{ab}}$ & $0.86 \pm 0.04^{\mathrm{ab}}$ & $0.90 \pm 0.02^{\mathrm{a}}$ & $0.83 \pm 0.02^{\mathrm{b}}$ & 0.039 \\
$\mathrm{PER}^{6}$ & $2.29 \pm 0.02^{\mathrm{ab}}$ & $2.27 \pm 0.11^{\mathrm{ab}}$ & $2.17 \pm 0.05^{\mathrm{b}}$ & $2.37 \pm 0.04^{\mathrm{a}}$ & 0.028 \\
$\mathrm{CF}^{7}$ & $1.61 \pm 0.05$ & $1.50 \pm 0.05$ & $1.51 \pm 0.03$ & $1.63 \pm 0.09$ & 0.060 \\
$\mathrm{Fl}^{8}\left(\%\right.$ day $\left.^{-1}\right)$ & $1.67 \pm 0.01$ & $1.65 \pm 0.04$ & $1.64 \pm 0.03$ & $1.71 \pm 0.01$ & 0.051 \\
\hline
\end{tabular}

Diseño experimental 2: formulación de las dietas Cuatro dietas formuladas isoproteícas (50\%) e isolipídicas (12\%). Las cuatro dietas se formularon con una proporción 2:1 de PBM: FM y $24 \%$ de SBM. La dieta control no contiene ningún compuesto funcional (SBM), la segunda dieta tiene un $1.5 \%$ de glutamina (GLN), la tercera un $1 \%$ de fructano de agave (AGA) y la cuarta dieta una combinación de glutamina y fructano (GLN+AGA).

\section{Organismos y facilidades}

Los juveniles se obtuvieron del Laboratorio de peces marinos del Centro de Investigación Científica y de Educación Superior de Ensenada. En ambos experimentos se colocaron aleatoriamente cinco peces en 12 tanques y se mantuvieron en un sistema de recirculación cerrado con una renovación del $5 \%$. La temperatura se mantuvo a $24 \pm 0.3^{\circ} \mathrm{C}$, el oxígeno a $6.7 \pm 0.7 \mathrm{mg} \mathrm{L}-$ ${ }^{1}$, salinidad de $35.3 \pm 0.2 \%$. Los peces fueron alimentados 3 veces al día a saciedad aparente por 44 días para el experimento 1 y 56 días para el experimento 2 .

Desempeño zootécnico y análisis histológico Se siguió la metodología descrita por FuentesQuesada et al., (2018; 2020).

\section{Resultados y discusión}

Los peces alimentados con $2 \%$ de AGA presentaron un mejor desempeño al tener un peso final, TGC (thermal growth coefficient, por sus siglas en inglés), WG (weight gain, por sus siglas en inglés), RWG (relative weight gain, por sus siglas en inglés), FCR (feed convertion rate, por sus siglas en inglés) y PER (protein efficiency ratio, por sus siglas en inglés) que las dieta SBM sin prebiótico. Estos resultados son similares a los encontrados en otras especies (Zhou et al., 2010; Ortíz et al., 2012). 
Figura 1. Imágenes del intestino distal de totoaba alimentada por 44 días con las dietas experimentales.
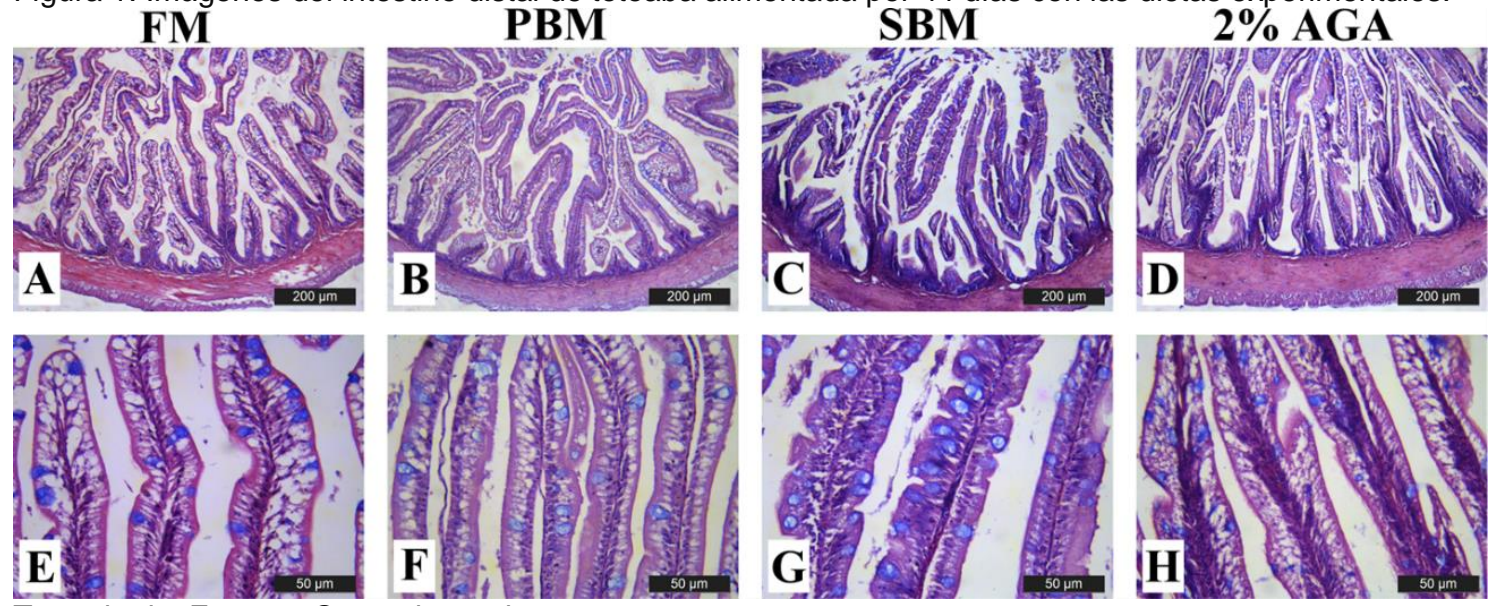

Tomado de: Fuentes-Quesada et al., 2020.

Los peces alimentados con la dieta SBM presentan las características asociadas a procesos de enteritis, como infiltración de leucocitos, disminución de vacuolas supranucleares, hiperplasia e hipertrofia de las células de goblet, y reducción del borde de cepillo. Sin embargo, la adición de $2 \%$ de fructano redujo el efecto negativo de la inclusión de harina de soya. Estos efectos beneficiosos de los prebióticos han sido reportados en otras especies (Zhou et al., 2010; Torrecillas et al., 2013; Bai et al., 2017).

Experimento 2.

Tabla 2. Crecimiento y utilización del alimento de totoaba a los 56 días de alimetnación

\begin{tabular}{|c|c|c|c|c|c|}
\hline & SBM & GLN & AGA & GLN+AGA & $P$ value \\
\hline Initial weight (g) & $167.9 \pm 5.5$ & $167.5 \pm 3.4$ & $167.5 \pm 3.6$ & $167.8 \pm 5.4$ & 0.999 \\
\hline Final weight (g) & $315.9 \pm 10.0^{b}$ & $345.4 \pm 9.8^{a}$ & $322.7 \pm 13.5^{b}$ & $329.4 \pm 13.3^{\mathrm{ab}}$ & 0.015 \\
\hline $\mathrm{TGC}^{1}$ & $0.99 \pm 0.01^{b}$ & $1.15 \pm 0.08^{\mathrm{a}}$ & $1.03 \pm 0.06^{b}$ & $1.07 \pm 0.05^{\mathrm{ab}}$ & 0.008 \\
\hline$W^{2}(g)$ & $147.9 \pm 4.7^{b}$ & $177.9 \pm 13.2^{a}$ & $155.1 \pm 12.1^{b}$ & $161.6 \pm 9.6^{a b}$ & 0.007 \\
\hline $\mathrm{RWG}^{3}(\%)$ & $88.1 \pm 1.4^{b}$ & $106.3 \pm 10.0^{\mathrm{a}}$ & $92.6 \pm 6.8^{b}$ & $96.3 \pm 4.9^{\mathrm{ab}}$ & 0.009 \\
\hline $\mathrm{DWG}^{4}(\mathrm{~g})$ & $2.64 \pm 0.08^{b}$ & $3.18 \pm 0.24^{\mathrm{a}}$ & $2.77 \pm 0.22^{b}$ & $2.89 \pm 0.17^{\mathrm{ab}}$ & 0.008 \\
\hline $\mathrm{FCR}^{5}$ & $0.92 \pm 0.02^{\mathrm{a}}$ & $0.84 \pm 0.04^{b}$ & $0.90 \pm 0.04^{\mathrm{a}}$ & $0.90 \pm 0.02^{\mathrm{ab}}$ & 0.014 \\
\hline $\mathrm{PER}^{6}$ & $2.17 \pm 0.05^{b}$ & $2.40 \pm 0.12^{\mathrm{a}}$ & $2.21 \pm 0.11^{b}$ & $2.22 \pm 0.05^{b}$ & 0.014 \\
\hline $\mathrm{PPV}^{7}$ & $0.38 \pm 0.01^{b}$ & $0.43 \pm 0.02^{\mathrm{a}}$ & $0.39 \pm 0.02^{b}$ & $0.39 \pm 0.01^{b}$ & 0.011 \\
\hline $\mathrm{CF}^{7}$ & $1.39 \pm 0.03$ & $1.43 \pm 0.05$ & $1.39 \pm 0.04$ & $1.37 \pm 0.01$ & 0.311 \\
\hline $\mathrm{FI}^{8}\left(\% \mathrm{day}^{-1}\right)$ & $1.01 \pm 0.04$ & $1.03 \pm 0.02$ & $1.01 \pm 0.06$ & $1.04 \pm 0.01$ & 0.301 \\
\hline
\end{tabular}

Los peces suplementados con glutamina presentaron un desempeño significativamente mayor que el resto de los tratamientos. Los beneficios de la glutamina en la salud intestinal en peces como mayor altura de los enterocitos e incremento de la longitud de los pliegues mucosos y de la densidad de las microvellosidades ha sido reportado en varias especies (Qiyou et al., 2011; Pohlenz et al., 2012; Gu et al., 2017) lo cual puede explicar el mejor crecimiento de los peces alimentado con glutamina.

\section{Referencias}

Bai, N. Gu, M. Xu, X. Xu and B. Krogdahl, Å. (2017). Protective effects of mannan oligosaccharides on turbot Scophthalmus maximus suffering from soy enteropathy, Aquaculture, 476: 141-151. https://doi.org/10.1016/j.aquaculture.2017.04.005

Bonvini, E., Bonaldo, A., Mandrioli, L., Sirri, R., Dondi, F., Bianco, C., Fontanillas, R., Mongile, F., Gatta, P. P. and Parma, L. (2018). Effects of feeding low fishmeal diets with increasing soybean meal levels on 
growth, gut histology and plasma biochemistry of sea bass. Animal, 12(5): 923-930. https://doi.org/10.1017/S1751731117002683

Chen, Z., Liu, Y., Li, Y., Yang, P., Hu, H., Yu, G., Ai, Q., Xu, W., Zhang, W. and Zhang, Y. (2018). Dietary arginine supplementation mitigates the soybean meal induced enteropathy in juvenile turbot, Scophthalmus maximus L. Aquac Res. 49:1535-1545. https://doi.org/10.1111/are.13608

Fuentes-Quesada, J. P., and Lazo, J. P. (2018). The effect of lipid type on lipid digestion enzymes during larval development of the California halibut, Paralichthys californicus. Aquaculture, 488:49-60. https://doi.org/10.1016/j.aquaculture.2018.01.018.

Fuentes-Quesada, J. P., Cornejo-Granados, F., Mata-Sotres, J. A., Ochoa-Romo, J. P., Rombenso, A. N., Guerrero-Rentería, Y., Lazo, J. P., Pohlenz, C., Ochoa-Leyva, A. and Viana, M. T. (2020). Prebiotic agavin in juvenile totoaba, Totoaba macdonaldi diets, to relieve soybean meal-induced enteritis: Growth performance, gut histology and microbiota. Aquaculture Nutrition, 26(6): 2115-2134. https://doi.org/10.1111/anu.13151

Gu, M., Bai, N., Zhang, Y. and Krogdahl, Å. (2016). Soybean meal induces enteritis in turbot Scophthalmus maximus at high supplementation levels. Aquaculture, 464: 286-295. https://doi.org/10.1016/j.aquaculture.2016.06.035

Gu, M., Bai, N., Xu, B., Xu, X., Jia Q. and Zhang, Z. (2017). Protective effect of glutamine and arginine against soybean meal-induced enteritis in juvenile turbot (Scophthalmus maximus). Fish \& Shellfish Immunology, 70: 95-105. https://doi.org/10.1016/i.fsi.2017.08.048

Guerreiro, I., Oliva-Teles, A. and Enes, P. (2017). Prebiotics as functional ingredients: focus on Mediterranean fish aquaculture Rev. Aquacult., 10 (4): 1-33. https://doi.org/10.1111/raq.12201

Krogdahl, Å., Penn, M., Thorsen, J., Refstie, S. and Bakke, A.M. (2010). Important antinutrientes in plant feedstuffs for aquaculture: an update on recent findings regarding responses in salmonids. Aquac. Res. 41(39): 333-344. https://doi.org/10.1111/j.1365-2109.2009.02426.x

Øverland, M., Mydland, L. T. and Skrede, A. (2019). Marine macroalgae as sources of protein and bioactive compounds in feed for monogastric animals. J. Sci. Food Agric., 99(1): 13-24. https://doi.org/10.1002/jsfa.9143

Ortiz, L. T., Rebole, A., Velasco, S., Rodriguez, M. L., Trevino, J., Tejedor, J. L. and Alzueta, C. (2012). Effects of inulin and fructooligosaccharide on growth performance, body composition and intestinal microbiota of farmed rainbow trout (Oncorhynchus mykiss). Aquaculture Nutrition, 19 (4): 475-482. https://doi.org/10.1111/j.1365-2095.2012.00981.x

Pelusio, N. F., Rossi, B., Parma, L., Volpe, E., Ciulli, S., Piva, A., D'Amico, F., Scicchitano, D., Candela, M., Gatta, P. P., Bonaldo, A. and Grilli, E. (2020). Effects of increasing dietary level of organic acids and nature-identical compounds on growth, intestinal cytokine gene expression and gut microbiota of rainbow trout (Oncorhynchus mykiss) reared at normal and high temperature. Fish \& Shellfish Immunology, 107 (part A): 324-335. http://dx.doi.org/10.1016/j.fsi.2020.10.021

Pereira, R. T., Rosa, P. V. and Gatlin III, D. M. (2017). Glutamine and arginine in diets for Nile tilapia: Effects on growth, innate immune responses, plasma amino acid profiles and whole-body composition. Aquaculture, 473, 135-144. https://doi.org/10.1016/j.aquaculture.2017.01.033

Pohlenz, C., Buentello, A., Bakke, A.M. and Gatlin III, D.M. (2012). Free dietary glutamine improves intestinal morphology and increases enterocyte migration rates, but has limited effects on plasma amino acid profile and growth performance of channel catfish Ictalurus punctatus. Aquaculture, 370-371: 32-39. https://doi.org/10.1016/j.aquaculture.2012.10.002

Qiyou, X., Qing, Z., Hong, X., Chang'an, W. and Dajiang, S. (2011). Dietary glutamine supplementation improves growth performance and intestinal digestion/absorption ability in young hybrid sturgeon (Acipenser schrenckii female $\times$ Huso dauricus male). J. Appl. Ichthyol, 27: 721-726. https://doi.org/10.1111/j.14390426.2011.01710.x

Torrecillas, S., Makol, A., Betancor, M. B., Montero, D., Caballero, M. J. and Sweetman, J. and Izquierdo, M.(2013). Enhanced intestinal epithelial barrier health status on European sea bass (Dicentrarchus labrax) fed mannan oligosaccharides. Fish \& Shellfish Immunology, 34(6): 1485-1495. https://doi.org/10.1016/i.fsi.2013.03.351

Zhou, Q. C., Buentello J. A. and Gatlin III., D. M. (2010). Effects of dietary prebiotics on growth performance, immune response and intestinal morphology of red drum (Sciaenops ocellatus). Aquaculture, 309(1-4): 253-257. https://doi.org/10.1016/j.aquaculture.2010.09.003 